\title{
Nitrogen-containing bisphosphonates are associated with reduced risk of pneumonia in patients with hip fracture
}

Chor-Wing Sing, $\mathrm{PhD}^{1}$, Douglas P. Kiel, MD, MPH${ }^{4}$, Richard B. Hubbard, $\mathrm{PhD}^{5}$, Wallis C.Y. Lau, $\mathrm{PhD}^{1,6}$, Gloria H.Y. Li, $\mathrm{PhD}^{1}$, Annie W.C. Kung, $\mathrm{MD}^{2}$, Ian C.K. Wong, $\mathrm{PhD}^{1,6}$, Ching-Lung Cheung, $\mathrm{PhD}^{1,2,3}$

${ }^{1}$ Department of Pharmacology and Pharmacy, ${ }^{2}$ Department of Medicine, ${ }^{3}$ Centre for Genomic Sciences, Li Ka Shing Faculty of Medicine, The University of Hong Kong, Pokfulam, Hong Kong.

${ }^{4}$ Hinda and Arthur Marcus Institute for Aging Research, Hebrew SeniorLife and Department of Medicine Beth Israel Deaconess Medical Center and Harvard Medical School, Boston, USA. ${ }^{5}$ Division of Epidemiology and Public Health, School of Medicine, University of Nottingham, Nottingham, United Kingdom. ${ }^{6}$ Research Department of Practice and Policy, UCL School of Pharmacy, London, United Kingdom.

\section{Correspondence and reprint requests:}

Ching-Lung Cheung, $\mathrm{PhD}$

Dept of Pharmacology and Pharmacy, 21 Sassoon Road, Pokfulam, Hong Kong Email: lung1212@hku.hk

Tel: +852-3917-9462 Fax: +852-2816-2095

Running title: Bisphosphonates reduced risk of pneumonia

Word count: 211 (Abstract); 3117 (Main text) 


\section{Disclosure}

All authors have completed and submitted the ICMJE Form for Disclosure of Potential Conflicts of Interest. Dr. Cheung and Prof Wong report receipt of research funding from Amgen, the Hong Kong Research Grants Council, and the Hong Kong Health and Medical Research Fund. Prof Wong has also received funding from Pfizer. BMS, Bayer, Novartis and Janssen. Dr. Kiel has received stipends for serving on a scientific advisory board for Solarea Bio, has received grants to his institution from the Dairy Council and Radius Health. He receives royalties for publication from Wolters Kluwer for UpToDate. No other disclosures were reported. 


\begin{abstract}
Objective To study the risk of pneumonia and pneumonia mortality among patients receiving nitrogen-containing bisphosphonates (N-BPs), non-N-BP anti-osteoporosis medications, and no anti-osteoporosis medications after hip fracture.
\end{abstract}

Methods This is a historical cohort study using a population-wide database. Patients with first hip fracture during 2005-2015 were identified and matched by time-dependent propensity score. The cohort was followed until December 312016 to capture any pneumonia and pneumonia mortality. Hazard ratios (HR) and 95\% confidence intervals (CI) were estimated using Cox-proportional hazards regression. Absolute risk difference (ARD) and number needed to treat (NNT) were calculated.

Results This study identified 54,047 patients with hip fracture. Of these, 4,041 patients who received N-BPs and 11,802 without anti-osteoporosis medication were propensity score matched. N-BPs were associated with a significantly lower risk of pneumonia compared with no treatment (6.9 vs 9.0 per 100 person-years; HR 0.76, 95\% CI 0.70-0.83), resulting in an ARD of 0.02 and NNT of 46. A similar association was observed with pneumonia mortality (HR $0.65,95 \%$ CI $0.56-$ 0.75). When N-BPs were compared with non-N-BP anti-osteoporosis medications, the association remained significant.

Conclusions N-BPs were associated with lower risks of pneumonia and pneumonia mortality. Randomized Control Trials are now required to determine whether N-BPs, non-vaccine based medications, can reduce pneumonia incidence in high risk groups.

Keywords: antiresorptives, osteoporosis, epidemiology, general population studies 


\section{Introduction}

Nitrogen-containing bisphosphonates (N-BPs) are widely used in the treatment of postmenopausal osteoporosis, with alendronate being the first-line medication in many countries. Early studies have suggested potential beneficial effects of alendronate and related N-BPs on the lung. A pharmacokinetic study showed the highest concentration of alendronate in the trachea among all non-bone tissues studied and it was retained in the trachea $>60$ days after a single or 7-day repeated intravenous administration. ${ }^{1}$ Alendronate was still detected in the trachea with a concentration of $607 \mathrm{ng} / \mathrm{ml}$ (vs $1370 \mathrm{ng} / \mathrm{ml}$ detected in vertebra) seventy-two hours after oral ingestion. ${ }^{2}$ Moreover, alendronate targets the same pathway as statins. A previous animal study and a recent randomized controlled trial (RCT) showed that statins possess immunomodulatory effects ${ }^{3}$ and improve pneumonia survival. $^{4}$

Based on of the above evidence, we hypothesized that N-BPs could protect humans from pneumonia and its associated mortality. To test this hypothesis, we conducted a real-world population-based propensity score (PS) matched cohort study in hip fracture patients, in which

pneumonia was the leading cause of death. ${ }^{5}$ The risk of incident pneumonia and pneumonia mortality in hip fracture patients receiving N-BPs, compared with no anti-osteoporosis medication or non-N-BP anti-osteoporosis medications, was studied.

\section{Materials and Methods}

\section{Data source}


Data was collected from the Clinical Data Analysis and Reporting System (CDARS), an electronic medical database managed by the Hong Kong Hospital Authority (HKHA). HKHA is a public healthcare provider, serving $>80 \%$ of hospital admissions in Hong Kong. CDARS is a centralized database developed for research and audit. It includes anonymized records of demographics, admission, prescription, diagnosis, procedures, laboratory tests results, and deaths. The database has been widely used in population-based studies ${ }^{5,6}$ and specifically validated for bone fractures studies. $^{7}$

\section{Study cohort}

We identified a historical study cohort using CDARS. Patients who aged $\geq 50$ years and were admitted via an emergency room between January 12005 and December 31 2015, with an incident hip fracture (ICD-9 code 820.XX) were included. To reduce selection bias and/or competing risk of death, we excluded patients who had i) previous exposure to anti-osteoporosis medications since 1993 when data were first available in CDARS; ii) prolonged length of stay (LOS) after hip fracture, defined as $>60$ days according to the general LOS of patients with hip fracture in Hong $\mathrm{Kong}^{8}$, as patients with prolonged LOS might be physically unfit to receive anti-osteoporosis medications; or iii) history of cancer since bone targeting agents are often prescribed. All patients in the study cohort were followed until December 312016 (end of study) to allow at least one year of follow-up.

\section{Exposure and outcomes}


Patients were classified as "N-BPs-exposed" if they had a prescription record of any N-BPs, including alendronate, ibandronate, risedronate, and zoledronate, before the end of study. Bisphosphonates can accumulate in the skeleton ${ }^{9}$ and their treatment effects can last for 10 years or longer. A prospective study showed that alendronate can sustain its long-term residual effects of reduction in bone turnover up to 7 years after treatment withdrawal. ${ }^{10} \mathrm{~A}$ recent open-label extension study of a randomized trial also suggests that 2 doses of $5 \mathrm{mg}$ zoledronate administered about 6 years apart can prevent bone loss over almost 11 years. ${ }^{11}$ Due to the above characteristics and to emulate the "intention-to-treat" principle, the patients were considered exposed to the drug if they received any treatment with N-BPs until the end of follow-up.

In the primary analysis, we compared patients treated with N-BPs (N-BPs-exposed) to patients without any anti-osteoporosis medication treatment (non-exposed). In the secondary analysis, we compared patients treated with N-BPs to patients treated with non-N-BP anti-osteoporosis medications with different mechanism-of-actions, namely denosumab, raloxifene, salcatonin, strontium ranelate, and teriparatide.

The outcomes of interest were pneumonia incidence and death (pneumonia mortality). Diagnosis of pneumonia was defined as in-patient/out-patient records coded with ICD-9 codes 480-487.0. Pneumonia mortality was defined by a cause of death with ICD-10 codes J12-J18.

\section{Propensity Score matching}


Propensity scores (PS) were used to reduce potential confounding by non-randomized treatment allocation. ${ }^{12}$ Propensity score was defined as a conditional probability of receiving certain treatment given a patient's characteristics. Details and covariates used to estimate PS are provided in Supplementary Method and Supplementary Table S1. Immortal time bias that favours the treatment group might arise due to the differences in follow-up start day between patients in nonexposed and N-BPs-exposed groups. ${ }^{13}$ To address the bias, we adopted a time-dependent PS matching approach, where N-BPs-exposed patients were matched with patients who were not yet exposed to N-BPs at the particular time point, allowing the comparison groups to be followed from the same starting point. ${ }^{14}$ Details of the time-dependent PS matching are described in the Supplementary Methods. To reduce any unmeasured confounding, PS trimming was performed before matching, in which patients with treatment status contrary to the prediction, i.e. patients with PS $<5^{\text {th }}$ percentile of treated or $>95^{\text {th }}$ percentile of untreated, were excluded. ${ }^{15}$

Given that the exposure status is time-dependent, a patient was considered as non-exposed until the initial prescription of N-BP. In the primary analysis, each N-BPs-exposed patient was matched with up to three non-exposed patients using sequential greedy matching ${ }^{16}$ with a caliper of 0.2 standard deviations (SD), without replacement. In the secondary analysis, each patient in the nonN-BP anti-osteoporosis medication group was matched with up to three patients in the N-BPsexposed group, without replacement, since more patients were treated with N-BPs than non-N-BP anti-osteoporosis medications. To assess the quality of matching, the absolute standardized differences (ASD) in covariates between treatment groups were estimated. ASD $<0.1$ was considered as well-balanced matching. ${ }^{17}$ Any covariate with $\mathrm{ASD} \geq 0.1$ was further adjusted in the regression analysis. 
Patients were followed from the index date until the occurrence of a pneumonia event, switch to another anti-osteoporosis medication, death, or end of study, whichever occurred first. The index date in N-BP-exposed patients was the date of first prescription while the index date in nonexposed patients was matched with N-BPs-exposed patients.

\section{Statistical analysis}

Continuous variables were presented as mean $\pm \mathrm{SD}$ and categorical variables as frequency (percentage). Incidence rates per 100 person-years and 95\% confidence intervals (CIs) were estimated using Poisson distribution. Time-to-event analysis was used to evaluate the association of N-BPs with outcomes. Hazard ratios (HRs) and 95\% CIs were estimated using Cox proportional hazards regression models stratified on the matched pairs. Kaplan-Meier curves comparing treatment groups were plotted and tested using a stratified log-rank test on matched pairs. The

absolute risk difference (ARD) between treatment groups was estimated using the formula (incidence rate ratio -1) $\mathrm{x}$ incidence rate of event in non-exposed group, where incidence rate ratio was interpreted as HR, given the large sample size. The number needed to treat (NNT) was calculated as the reciprocal of ARD.

\section{Additional analyses}

Subgroup analyses were performed by sex and history of pneumococcal/seasonal influenza vaccination. Sensitivity analyses were conducted to detect any residual and unmeasured confounding. First, we excluded patients receiving late treatment (start of first treatment>180 days 
from the time of discharge from hip fracture). Since a longer time from hip fracture is associated with a lower pneumonia risk, patients with delayed treatment were excluded to prevent bias. The 180 days cut-off was used because mortality of hip fracture stabilized after 180 days. ${ }^{5}$ Second, we repeated the analysis in the unmatched cohort using inverse probability of treatment weighting (IPTW). PS-matching excluded unmatched subjects in the analysis, limiting the sample size and generalizability of the study. Conversely, IPTW retains all subjects in the study cohort and overcomes the limitation in PS-matching. Details of IPTW and calculation of the weights using PS were discussed elsewhere. ${ }^{18}$ In this study, we conducted IPTW using stabilized weights with truncation at 5\%. Finally, the E-value ${ }^{19,20}$ was also computed to further evaluate the robustness of the findings to unmeasured confounding. ${ }^{21}$

$\mathrm{R}$ was used for all statistical analyses. A two-sided $\mathrm{p}$-value $<0.05$ was considered significant.

\section{Results}

\section{Baseline characteristics}

We identified 54,047 patients from the database. Among the 43,349 patients included after screening (Figure 1), 6,467 (14.9\%) were prescribed anti-osteoporosis medication by the end of study. In the primary analysis, 4,041 N-BPs-exposed patients were matched with 11,802 nonexposed patients. The covariates were well-matched ( $\mathrm{ASD}<0.1)$ except for the year of index date, frequency of in-patient admissions, and the use of anticoagulants and nonsteroidal antiinflammatory drugs in the past 180 days (Table 1), which were later adjusted in the analysis. In the secondary analysis, 1,284 N-BP exposed patients were matched with 507 non-N-BP anti- 
osteoporosis medications exposed patients (166 on strontium ranelate, 161 on salcatonin, 135 on denosumab, 38 on teriparatide, and 7 on raloxifene). All covariates were well-matched (Supplementary Table S2). The Kaplan-Meier curves between treatment groups showed significant difference in the pneumonia events (Figure 2).

\section{N-BPs and risk of pneumonia}

The median follow-up time was 2.7 years (interquartile range 1.3 to 5.1 years). The incidence of pneumonia was 9.0 and 6.9 per 100 patient-years for the non-exposed and N-BPs-exposed groups, respectively (Table 2). N-BPs were associated with a reduced risk of incident pneumonia (HR 0.76, 95\% CI 0.70 to 0.83 ; Table 2), with an ARD of 0.02 (95\% CI 0.02 to 0.03 ), and 46 (95\% CI 37 to 65) patients were required to treat to prevent one pneumonia. Similar significant findings were observed for alendronate exposure (Table 2).

No significant interaction was observed for N-BPs with gender and vaccination, and subgroup analyses revealed similar findings (Supplementary Table S3). In the sensitivity analyses (Supplementary tables S4), excluding late treatment and IPTW analysis revealed similar findings. The E-value for point estimate and confidence interval was 1.96 (1.7). 


\section{N-BPs and risk of pneumonia mortality}

The pneumonia mortality was 3.5 and 2.3 per 100 patient-years for the non-exposed and N-BPsexposed groups, respectively (Table 2). N-BPs were associated with a reduced risk of pneumonia mortality (HR $0.65,95 \%$ CI 0.56 to 0.75 ; Table 2). Similar significant findings were observed for alendronate exposure (Table 2). No significant interaction was observed for N-BP with gender and vaccination on pneumonia mortality (Supplementary Table S3). Similar results were observed in the subgroup (Supplementary Table S3) and sensitivity analyses (Supplementary Tables S4). The E-value for point estimate and confidence interval was 2.45 (2).

\section{N-BPs vs. non-N-BP anti-osteoporosis medications}

To avoid confounding by indication, non-N-BP anti-osteoporosis medications was considered as the comparator. Similarly, the association of N-BPs was significant (pneumonia: HR 0.68, 95\% CI 0.53 to 0.87 ; pneumonia mortality: HR $0.60,95 \%$ CI 0.41 to 0.89 ; Table 3 ). Using the IPTW method with maintained sample size, significant associations of N-BPs with reduced risk of pneumonia incidence and mortality were observed (pneumonia: HR $0.52,95 \%$ CI $0.44-0.61$; pneumonia mortality: HR 0.38, 95\% CI 0.3-0.47; Supplementary Table S4).

\section{Discussion}

This is the first real-world population-based study using a large electronic clinical database to examine the potential effect of N-BPs on post-hip fracture pneumonia risk. Patients prescribed N- 
BPs had a significantly reduced pneumonia risk and mortality when compared with those without any treatment or with non-N-BP anti-osteoporosis medications. The effect was robust in various sensitivity and subgroup analyses.

\section{Potential mechanisms}

This hypothesis-testing study was based on evidence suggesting that N-BPs may have similar effects on both alveolar macrophages and osteoclasts, cells that share the same lineage, and thus may influence the pathogenesis of pneumonia characterized by lung parenchyma inflammation. Alveolar macrophages play an important antibacterial role in defending against pneumonia by early phagocytosis of pathogens and subsequent induction of apoptosis to minimize inflammation. ${ }^{22}$ A clinical study showed that N-BP reduced macrophage lineage cells ${ }^{23}$ through the reduction of mcl-1 expressions in both macrophages ${ }^{24}$ and osteoclasts ${ }^{25}$, which might subsequently reduce inflammation. ${ }^{22}$ The pharmacology ${ }^{22,25}$ and pharmacokinetics ${ }^{1,2}$ of N-BPs also make it a potential drug for pneumonia as previously mentioned. N-BP (pamidronate) expands human $\mathrm{V} \gamma 9 \mathrm{~V} \delta 2 \mathrm{~T}$-cell populations in humanized mice, kills influenza -infected cells, inhibits in vitro influenza viral replication, and subsequently reduces the severity of influenza infection and the associated mortality. ${ }^{26}$ Moreover, both N-BPs and statins target the same pathway, and possess similar anti-inflammation and immunomodulatory effects. ${ }^{3,27}$ In a recent RCT, statins improved neutrophil function and hospital-free survival in pneumonia. ${ }^{4}$ Furthermore, it is also proposed that N-BP (zoledronate) could maintain physiological reserve, thus enhancing the ability to recover from acute illnesses. ${ }^{28}$ Together with the current study, this evidence suggested N-BPs as a new promising drug class in reducing risk of pneumonia and its associated mortality. 


\section{Comparison with other studies}

Our study is in agreement with a post-hoc analysis ${ }^{28}$ of HORIZON Recurrent Fracture Trial, which demonstrated that zoledronate reduced pneumonia mortality by $\sim 50 \%$ after a mean follow-up of 2 years. Similarly, we observed HRs of 0.65 in N-BPs group at a 3-year (median) follow-up for pneumonia mortality. Although the post-hoc analysis of the RCT showed only a slightly lower incidence of pneumonia in the zoledronate group $(5.5 \%$ in zoledronate group vs. $5.6 \%$ in placebo group), such a discrepancy could be due to the highly selected patients in the HORIZON RCT, which excluded patients who were unwilling/unable to take oral bisphosphonate and had life expectancy $<1$ year as judged by the investigators. Patients with high risk of pneumonia might be excluded, leading to reduced statistical power. Moreover, the self-reported pneumonia incidence was subjected to under-diagnosis, loss of follow-up, and recall and misclassification bias, resulting in a bias towards the null hypothesis. Conversely, the larger sample size of the current study has increased the power to detect differences with statistical significance. Also, we used the clinical diagnosis of pneumonia by physicians, which is more accurate and less biased. It should be noted that our study included mainly alendronate-exposed patients (81.6\%); however given that alendronate and zoledronate have the same pharmacology with different potency ${ }^{29}$, it is possible that alendronate could have similar effects on reducing pneumonia, which is supported by our findings. Further studies investigating the link between alendronate and pneumonia are warranted.

Previous RCTs and observational studies have suggested $10 \%$ to $60 \%$ reduction of all-cause mortality by bisphosphonates ${ }^{30-32}$ Among these studies, only a few reported the cause of death ${ }^{31}$ 
or the risk of pneumonia mortality ${ }^{32}$. Apart from the post-hoc analysis of the HORIZON Recurrent Fracture Trial as previously mentioned, a prospective study involving 78 bisphosphonates users (63 on alendronate) and 1,923 non-users has reported a reduced risk of death due to infection (including pneumonia) but the association did not reach statistical significance (HR 0.64, 95\%CI $0.35-1.19, \mathrm{p}=0.16) .{ }^{32}$ Indeed, the point estimate reported in this study is similar in magnitude to our finding; however the lack of statistical significance possibly could be due to the lack of power with a small sample. Therefore, more studies on the effect of N-BPs on cause-specific mortality are warranted. On the other hand, a recent meta-analysis of RCTs reported no association between bisphosphonates and overall mortality. ${ }^{33}$ This meta-analysis of 38 RCTs included patients with osteoporosis, osteopenia, and osteoporotic fractures. Indeed, if RCTs of patients with osteoporotic fractures was selected, a reduced risk of mortality (pooled risk ratio $0.79 ; 95 \%$ CI $0.65-0.95 ; \mathrm{I}^{2}$ $=0 \%$ ) would be observed. Although the pooled risk ratio was largely driven by one RCT which studied zoledronate, as mentioned previously, it is possible that alendronate would have similar effects as zoledronate. In addition, there is a longstanding debate that RCTs excluding patients with chronic health conditions result in a healthier cohort that is unrepresentative of the real-world setting. ${ }^{34}$ Bisphosphonate studies are susceptible to such bias because in clinical settings, the drugs are commonly used in old patients with multiple chronic diseases. The effect of bisphosphonates in reducing mortality might be more pronounced in higher-risk groups e.g. patients with osteoporotic fractures, or critically ill patients ${ }^{35}$ than lower-risk groups. We, therefore, suggest the inclusion of high-risk patients in future anti-osteoporosis treatment RCTs.

Since frail patients would be less likely to receive anti-osteoporosis medications after hip fracture, bias in the prescribing of treatment could exist. As shown in Table 1, N-BPs-exposed patients in 
the pre-matched cohort generally had less comorbidity compared with the non-exposed group. We, therefore, used patients receiving non-N-BP anti-osteoporosis medications as a comparator. A significant reduced risk of pneumonia and pneumonia mortality was still observed. On the other

hand, patients receiving medication treatment could be wealthier and thus could get a better medical care, resulting in a lower risk of infections and mortality. Nonetheless, such bias should be minimal because the costs of N-BPs are highly subsidized in Hong Kong. We included non-N$\mathrm{BP}$ anti-osteoporosis medications as comparators, and the costs of these drugs like teriparatide and denosumab are even higher than N-BPs. In addition, the analyses were repeated using injury or trauma hospitalization as a negative control outcome. ${ }^{36}$ No significant association was observed (Supplementary Table S5), suggesting minimal confounding bias.

\section{Clinical implication}

Currently, vaccination is the only medication that can prevent pneumonia. In our subgroup analysis, we showed that N-BPs were associated with reduced pneumonia risk and mortality, regardless of the vaccination status. N-BPs could therefore confer additional protection against pneumonia to compensate for the reported shortage and low acceptance of vaccine, as well as the high cost of large-scale vaccination program. Drug repositioning of N-BPs as a pneumonia-prevention drug, especially in high-risk groups (e.g. patients with osteoporosis), may be of public health importance. The multiple benefits of using N-BPs in hip fracture patients may help to promote the use of these medications, especially in light of the fact that anti-osteoporosis medications are under-utilized. ${ }^{37}$ Given the potential importance of N-BPs, further investigation or a RCT is warranted. 


\section{Strengths and limitations}

Our study has several strengths. A clinical database capturing most records with high validity ${ }^{38}$ was used to conduct this population-based study to provide ample power for association detection. We also used non-N-BP anti-osteoporosis medications as a comparator to minimize confounding by indication. The finding is likely generalizable to hip fracture patients, who are susceptible to pneumonia.

Limitations of the study included the unavailability of data regarding infection type. There might also be competing risk of death. However, such effect should be minimal since pneumonia is the top leading cause of death and similar results were observed using competing risk regression (data not shown). In addition, there might be potential residual confounding. Nonetheless, the calculated E-values of incident pneumonia and pneumonia mortality were greater than any risk factors included in the PS model (Supplementary Table S6), suggesting the presence of unmeasured confounding that could overcome the effect of N-BPs treatment observed was unlikely. Moreover, our previous study has revealed association between N-BP and reduced risk of myocardial infarction $^{5}$ and the association was subsequently observed in a large-scale RCT of zoledronate. ${ }^{39}$ Such external and independent validation in a RCT provided a strong support for the validity and causality of our findings. Besides, further studies are required to determine if the results are generalizable to other disease groups.

\section{Conclusion}


Among patients with hip fracture, the use of N-BPs was associated with a lower risk of pneumonia and associated mortality when compared to non-N-BP anti-osteoporosis or no anti-osteoporosis medication use. Future RCTs may be warranted to further validate the findings.

Contributors: C.W.S., D.P.K., R.B.H., C.L.C. designed the study. C.W.S. and C.L.C. collected data and conducted data analysis. C.W.S., D.P.K., R.B.H., W.C.L., A.W.K., I.C.W., C.L.C. interpret the data. C.W.S. and C.L.C. drafted the manuscript. C.W.S., D.P.K., R.B.H., W.C.L., G.H.L., A.W.K., I.C.W., C.L.C. revised the manuscript. All authors approved final version of manuscript. C.L.C. takes responsibility for the integrity of the data analysis.

\section{Acknowledgements: None}

\section{Funding: None}

Ethical approval: The study protocol was approved by the institutional review boards of the University of Hong Kong and Hong Kong Hospital Authority (HKHA); Reference: UW17-364.

\section{References}

1. Mochizuki T, Nishimura S, Okabe K, et al. Metabolic fate of 4-amino-1-hydroxybutylidene-1, 1bisphosphonate (alendronate) (II): plasma concentration, distribution and excretion after repeated intravenous administration to 7 -week old rats and after single intravenous administration to 30-week old rats, and transfer into the fetus and milk in rats. . Drug Metab Pharmacokinet. 1995;10(2):174-189.

2. Hisaka A, Takenaga N, Hara K, et al. Absorption, Distribution, Excretion, and Sex Differences in the Disposition of the Antiosteolytic Agent, Alendronate after Oral Administration in Rats. Drug Metab Pharmacokinet. 1998;13(3):252-258.

3. Greenwood J, Steinman L, Zamvil SS. Statin therapy and autoimmune disease: from protein prenylation to immunomodulation. Nat Rev Immunol. 2006;6(5):358-370.

4. Sapey E, Patel JM, Greenwood H, et al. Simvastatin Improves Neutrophil Function and Clinical Outcomes in Pneumonia: a Pilot Randomised Controlled Trial. Am J Respir Crit Care Med. 2019. 
5. Sing CW, Wong AY, Kiel DP, et al. Association of Alendronate and Risk of Cardiovascular Events in Patients With Hip Fracture. Journal of bone and mineral research : the official journal of the American Society for Bone and Mineral Research. 2018;33(8):1422-1434.

6. Wong $\mathrm{AY}$, Wong IC, Chui CS, et al. Association Between Acute Neuropsychiatric Events and Helicobacter pylori Therapy Containing Clarithromycin. JAMA internal medicine. 2016;176(6):828-834.

7. Lau WC, Chan EW, Cheung CL, et al. Association Between Dabigatran vs Warfarin and Risk of Osteoporotic Fractures Among Patients With Nonvalvular Atrial Fibrillation. Jama. 2017;317(11):1151-1158.

8. Lau TW, Fang C, Leung F. The effectiveness of a geriatric hip fracture clinical pathway in reducing hospital and rehabilitation length of stay and improving short-term mortality rates. Geriatric orthopaedic surgery \& rehabilitation. 2013;4(1):3-9.

9. Allen MR. Skeletal accumulation of bisphosphonates: implications for osteoporosis treatment. Expert Opin Drug Metab Toxicol. 2008;4(11):1371-1378.

10. Bagger YZ, Tanko LB, Alexandersen P, Ravn P, Christiansen C. Alendronate has a residual effect on bone mass in postmenopausal Danish women up to 7 years after treatment withdrawal. Bone. 2003;33(3):301-307.

11. Grey A, Horne A, Gamble G, et al. Ten Years of Very Infrequent Zoledronate Therapy in Older Women: An Open-Label Extension of a Randomized Trial. The Journal of Clinical Endocrinology \& Metabolism. 2020;105(4).

12. Austin PC. Balance diagnostics for comparing the distribution of baseline covariates between treatment groups in propensity-score matched samples. Stat Med. 2009;28(25):3083-3107.

13. Jones M, Fowler R. Immortal time bias in observational studies of time-to-event outcomes. J Crit Care. 2016;36:195-199.

14. Zeng C, Dubreuil M, LaRochelle MR, et al. Association of Tramadol With All-Cause Mortality Among Patients With Osteoarthritis. Jama. 2019;321(10):969-982.

15. Sturmer T, Wyss R, Glynn RJ, Brookhart MA. Propensity scores for confounder adjustment when assessing the effects of medical interventions using nonexperimental study designs. J Intern Med. 2014;275(6):570-580.

16. Austin PC. A comparison of 12 algorithms for matching on the propensity score. Stat Med. 2014;33(6):1057-1069.

17. Stuart EA, Lee BK, Leacy FP. Prognostic score-based balance measures can be a useful diagnostic for propensity score methods in comparative effectiveness research. J Clin Epidemiol. 2013;66(8 Suppl):S84-S90 e81.

18. Thoemmes F, Ong AD. A Primer on Inverse Probability of Treatment Weighting and Marginal Structural Models. Emerg Adulthood. 2016;4(1):40-59.

19. VanderWeele TJ, Ding P. Sensitivity Analysis in Observational Research: Introducing the E-Value. Ann Intern Med. 2017;167(4):268-274.

20. Mathur MB, Ding P, Riddell CA, VanderWeele TJ. Web Site and R Package for Computing Evalues. Epidemiology. 2018;29(5):e45-e47.

21. Haneuse S, VanderWeele TJ, Arterburn D. Using the E-Value to Assess the Potential Effect of Unmeasured Confounding in Observational Studies. Jama. 2019;321(6):602-603.

22. Preston JA, Bewley MA, Marriott HM, et al. Alveolar Macrophage Apoptosis-Associated Bacterial Killing Helps Prevent Murine Pneumonia. American Journal of Respiratory and Critical Care Medicine.0(ja):null.

23. D'Amelio P, Grimaldi A, Cristofaro MA, et al. Alendronate reduces osteoclast precursors in osteoporosis. Osteoporos Int. 2010;21(10):1741-1750. 
24. Tai TW, Chen CY, Su FC, et al. Reactive oxygen species are required for zoledronic acid-induced apoptosis in osteoclast precursors and mature osteoclast-like cells. Sci Rep. 2017;7:44245.

25. Sutherland KA, Rogers HL, Tosh D, Rogers MJ. RANKL increases the level of Mcl-1 in osteoclasts and reduces bisphosphonate-induced osteoclast apoptosis in vitro. Arthritis Res Ther. 2009;11(2):R58.

26. Tu W, Zheng J, Liu Y, et al. The aminobisphosphonate pamidronate controls influenza pathogenesis by expanding a gammadelta T cell population in humanized mice. J Exp Med. 2011;208(7):1511-1522.

27. Sasaki O, Imamura M, Yamazumi Y, et al. Alendronate attenuates eosinophilic airway inflammation associated with suppression of Th2 cytokines, Th17 cytokines, and eotaxin-2. J Immunol. 2013;191(6):2879-2889.

28. Colon-Emeric CS, Mesenbrink P, Lyles KW, et al. Potential mediators of the mortality reduction with zoledronic acid after hip fracture. Journal of bone and mineral research : the official journal of the American Society for Bone and Mineral Research. 2010;25(1):91-97.

29. Drake MT, Clarke BL, Khosla S. Bisphosphonates: mechanism of action and role in clinical practice. Mayo Clin Proc. 2008;83(9):1032-1045.

30. Bolland MJ, Grey AB, Gamble GD, Reid IR. Effect of osteoporosis treatment on mortality: a metaanalysis. J Clin Endocrinol Metab. 2010;95(3):1174-1181.

31. Center JR, Bliuc D, Nguyen ND, Nguyen TV, Eisman JA. Osteoporosis medication and reduced mortality risk in elderly women and men. J Clin Endocrinol Metab. 2011;96(4):1006-1014.

32. Sambrook PN, Cameron ID, Chen JS, et al. Oral bisphosphonates are associated with reduced mortality in frail older people: a prospective five-year study. Osteoporos Int. 2011;22(9):25512556.

33. Cummings SR, Lui LY, Eastell R, Allen IE. Association Between Drug Treatments for Patients With Osteoporosis and Overall Mortality Rates: A Meta-analysis. JAMA internal medicine. 2019.

34. Eisman JA, Geusens P, van den Bergh J. The Emperor's New Clothes: What Randomized Controlled Trials Don't Cover. Journal of bone and mineral research : the official journal of the American Society for Bone and Mineral Research. 2018;33(8):1394-1396.

35. Lee $\mathrm{P}, \mathrm{Ng} \mathrm{C}$, Slattery A, Nair P, Eisman JA, Center JR. Preadmission Bisphosphonate and Mortality in Critically III Patients. J Clin Endocrinol Metab. 2016;101(5):1945-1953.

36. Jackson LA, Jackson ML, Nelson JC, Neuzil KM, Weiss NS. Evidence of bias in estimates of influenza vaccine effectiveness in seniors. Int J Epidemiol. 2006;35(2):337-344.

37. Khosla S, Cauley JA, Compston J, et al. Addressing the Crisis in the Treatment of Osteoporosis: A Path Forward. Journal of bone and mineral research : the official journal of the American Society for Bone and Mineral Research. 2017;32(3):424-430.

38. Sing CW, Woo YC, Lee ACH, et al. Validity of major osteoporotic fracture diagnosis codes in the Clinical Data Analysis and Reporting System in Hong Kong. Pharmacoepidemiol Drug Saf. 2017;26(8):973-976.

39. Reid IR, Horne AM, Mihov B, et al. Fracture Prevention with Zoledronate in Older Women with Osteopenia. N Engl J Med. 2018;379(25):2407-2416. 


\section{Figure Legends}

Figure 1. Screening flow chart of the study cohort

Figure 2. Kaplan-Meier curves comparing treatment groups for a) incident pneumonia and b)

pneumonia mortality. ( $\mathrm{p}$-value tested with stratified log-rank test on matched pairs) 
Table 1. Baseline characteristics of the study population before and after propensity score matching, patients treated with N-BPs compared with no treatment

\begin{tabular}{|c|c|c|c|c|c|c|}
\hline & \multicolumn{3}{|c|}{ Pre-matched cohort } & \multicolumn{3}{|c|}{ Matched cohort } \\
\hline & Non-exposed & N-BPs exposed & ASD & Non-exposed & N-BPs exposed & ASD \\
\hline Subject, n (\%) & 37,542 & 5,807 & & 11,802 & 4,041 & \\
\hline Male, n (\%) & $12,178(32.4)$ & $1,229(21.2)$ & 0.26 & $2,791(23.6)$ & $928(23.0)$ & 0.02 \\
\hline Age, mean (SD) & $81.9(9.5)$ & $80.0(8.6)$ & 0.22 & $80.3(9.8)$ & $80.3(8.4)$ & $<0.01$ \\
\hline $50-69$ & $3,835(10.2)$ & $715(12.3)$ & & $1617(13.7)$ & $446(11.0)$ & \\
\hline $70-89$ & $25,844(68.8)$ & $4,458(76.8)$ & & $8314(70.4)$ & $3,131(77.5)$ & \\
\hline $90+$ & $7,863(20.9)$ & $634(10.9)$ & & $1871(15.9)$ & $464(11.5)$ & \\
\hline Year of index date, $\mathrm{n}(\%)$ & & & 0.66 & & & 0.31 \\
\hline 2005 & $3,048(8.1)$ & $46(0.8)$ & & $419(3.6)$ & $43(1.1)$ & \\
\hline 2006 & $3,325(8.9)$ & $95(1.6)$ & & $376(3.2)$ & $71(1.8)$ & \\
\hline 2007 & $3,323(8.9)$ & $272(4.7)$ & & $962(8.2)$ & $196(4.9)$ & \\
\hline 2008 & $3,555(9.5)$ & $350(6.0)$ & & $1,092(9.3)$ & $271(6.7)$ & \\
\hline 2009 & $3,252(8.7)$ & $584(10.1)$ & & $1,279(10.8)$ & $401(9.9)$ & \\
\hline 2010 & $3,201(8.5)$ & 808 (13.9) & & $1,272(10.8)$ & $485(12.0)$ & \\
\hline 2011 & $3,493(9.3)$ & $661(11.4)$ & & $1,154(9.8)$ & $472(11.7)$ & \\
\hline 2012 & $3,487(9.3)$ & $564(9.7)$ & & $1,023(8.7)$ & $396(9.8)$ & \\
\hline 2013 & $3,406(9.1)$ & $636(11.0)$ & & $1,126(9.5)$ & $445(11.0)$ & \\
\hline 2014 & $3,662(9.8)$ & $762(13.1)$ & & $1,407(11.9)$ & $493(12.2)$ & \\
\hline 2015 & $3,498(9.3)$ & $693(11.9)$ & & $1,367(11.6)$ & $532(13.2)$ & \\
\hline 2016 & $292(0.8)$ & $336(5.8)$ & & $325(2.8)$ & $236(5.8)$ & \\
\hline Admission hospital cluster, $\mathrm{n}(\%)$ & & & & & & 0.09 \\
\hline Hong Kong West Cluster & $2,842(7.6)$ & $409(7.0)$ & & $1,056(8.9)$ & $314(7.8)$ & \\
\hline Hong Kong East Cluster & $4,307(11.5)$ & $937(16.1)$ & & $1,925(16.3)$ & $596(14.7)$ & \\
\hline New Territories West Cluster & $4,562(12.2)$ & $307(5.3)$ & & $650(5.5)$ & $227(5.6)$ & \\
\hline New Territories East Cluster & $6,252(16.7)$ & $1,026(17.7)$ & & $2,252(19.1)$ & $842(20.8)$ & \\
\hline Kowloon West Cluster & $7,442(19.8)$ & $1,309(22.5)$ & & $2,588(21.9)$ & $844(20.9)$ & \\
\hline
\end{tabular}




\section{Kowloon East Cluster}

Kowloon Central Cluster

Nursing home residency, n (\%)

Seasonal influenza / Pneumococcal vaccination, n $(\%)$

Surgical operation for hip fracture, n $(\%)$

Frequency of healthcare service in past one year, mean (SD)

In-patient admission

Out-patient clinics

Intensive care units

Medical history, n (\%)

Coronary heart disease

Congestive heart failure

Arrhythmia and conduction disorders

Arterial disease

Hypertensive disease

Cerebrovascular disease

Chronic obstructive pulmonary disease

Other lung diseases

Diabetes

Hyperlipidemia

Obesity

Renal failure

Chronic liver disease

Osteoporosis

Fall

Other major fractures

Connective tissue disease

Dementia

Thyroid disorders

\begin{tabular}{|c|c|c|c|c|c|}
\hline 4,698 (12.5) & $1,339(23.1)$ & & $2,190(18.6)$ & $858(21.2)$ & \\
\hline 7,439 (19.8) & $480(8.3)$ & & $1,141(9.7)$ & $360(8.9)$ & \\
\hline $10,706(28.5)$ & 778 (13.4) & 0.38 & $1,426(12.1)$ & $518(12.8)$ & 0.02 \\
\hline 4,004 (10.7) & $1,020(17.6)$ & 0.20 & $1,683(14.3)$ & $637(15.8)$ & 0.04 \\
\hline $33,158(88.3)$ & $5,650(97.3)$ & 0.35 & $11,448(97.0)$ & $3,948(97.7)$ & 0.04 \\
\hline $1.9(3.3)$ & $1.5(2.0)$ & 0.13 & $1.6(1.5)$ & $1.4(1.1)$ & 0.16 \\
\hline $7.9(10.1)$ & $11.6(11.8)$ & 0.34 & $9.9(10.5)$ & $10.2(9.8)$ & 0.03 \\
\hline $0.02(0.13)$ & $0.01(0.12)$ & 0.01 & $0.02(0.13)$ & $0.01(0.10)$ & 0.05 \\
\hline 5,043 (13.4) & $602(10.4)$ & 0.10 & $1,276(10.8)$ & 390 ( 9.7) & 0.04 \\
\hline $4,147(11.0)$ & $445(7.7)$ & 0.12 & $908(7.7)$ & $287(7.1)$ & 0.02 \\
\hline 4,917 (13.1) & $592(10.2)$ & 0.09 & $1,261(10.7)$ & $403(10.0)$ & 0.02 \\
\hline $1,814(4.8)$ & $246(4.2)$ & 0.03 & $487(4.1)$ & $161(4.0)$ & 0.01 \\
\hline $16,216(43.2)$ & $2,584(44.5)$ & 0.03 & $5,037(42.7)$ & $1,669(41.3)$ & 0.03 \\
\hline 7,032 (18.7) & $872(15.0)$ & 0.10 & $1,732(14.7)$ & $610(15.1)$ & 0.01 \\
\hline $3,829(10.2)$ & $531(9.1)$ & 0.04 & 998 ( 8.5) & $328(8.1)$ & 0.01 \\
\hline $7,547(20.1)$ & $1,070(18.4)$ & 0.04 & $2,021(17.1)$ & 694 (17.2) & $<0.01$ \\
\hline $8,296(22.1)$ & $1,356(23.4)$ & 0.03 & $2,662(22.6)$ & 854 (21.1) & 0.03 \\
\hline $3,473(9.3)$ & $681(11.7)$ & 0.08 & $1,214(10.3)$ & $394(9.8)$ & 0.02 \\
\hline $102(0.3)$ & $23(0.4)$ & 0.02 & $44(0.4)$ & $10(0.2)$ & 0.02 \\
\hline $2,119(5.6)$ & $135(2.3)$ & 0.17 & $302(2.6)$ & $95(2.4)$ & 0.01 \\
\hline $304(0.8)$ & $35(0.6)$ & 0.03 & $88(0.7)$ & $24(0.6)$ & 0.02 \\
\hline $1,576(4.2)$ & 849 (14.6) & 0.36 & $551(4.7)$ & $224(5.5)$ & 0.04 \\
\hline $36,431(97.0)$ & 5,691 (98.0) & 0.06 & $11,504(97.5)$ & 3,957 (97.9) & 0.03 \\
\hline 4,408 (11.7) & 885 (15.2) & 0.10 & $1,584(13.4)$ & $560(13.9)$ & 0.01 \\
\hline $221(0.6)$ & $128(2.2)$ & 0.14 & $104(0.9)$ & $46(1.1)$ & 0.03 \\
\hline $3,416(9.1)$ & $236(4.1)$ & 0.20 & $392(3.3)$ & $140(3.5)$ & 0.01 \\
\hline $1,007(2.7)$ & $171(2.9)$ & 0.02 & $336(2.8)$ & 109 ( 2.7) & 0.01 \\
\hline
\end{tabular}




\begin{tabular}{|c|c|c|c|c|c|c|}
\hline \multicolumn{7}{|l|}{ Prescription in past 180 days, $\mathrm{n}(\%)$} \\
\hline Digoxin & $1,256(3.3)$ & $123(2.1)$ & 0.08 & $271(2.3)$ & $90(2.2)$ & 0.01 \\
\hline Loop diuretics & $5,074(13.5)$ & $571(9.8)$ & 0.12 & $1,241(10.5)$ & $377(9.3)$ & 0.04 \\
\hline Other diuretics & $2,797(7.5)$ & $401(6.9)$ & 0.02 & $841(7.1)$ & $305(7.5)$ & 0.02 \\
\hline Anti-arrhythmics class I and II & $668(1.8)$ & $69(1.2)$ & 0.05 & $197(1.7)$ & $43(1.1)$ & 0.05 \\
\hline Beta blockers & $7,977(21.2)$ & $1,218(21.0)$ & 0.01 & $2,538(21.5)$ & $823(20.4)$ & 0.03 \\
\hline $\begin{array}{l}\text { Angiotensin receptor blocker/ angiotensin } \\
\text { converting enzyme inhibitor/ renin inhibitor }\end{array}$ & $8,692(23.2)$ & $1,415(24.4)$ & 0.03 & $2,761(23.4)$ & $939(23.2)$ & $<0.01$ \\
\hline Nitrates & $3,973(10.6)$ & $460(7.9)$ & 0.09 & $953(8.1)$ & $309(7.6)$ & 0.02 \\
\hline Calcium channel blockers & $14,296(38.1)$ & $2,137(36.8)$ & 0.03 & $4,204(35.6)$ & $1,465(36.3)$ & 0.01 \\
\hline Peripheral vasodilators & $701(1.9)$ & $56(1.0)$ & 0.08 & $119(1.0)$ & $35(0.9)$ & 0.02 \\
\hline Anticoagulants & $2,375(6.3)$ & $316(5.4)$ & 0.04 & $766(6.5)$ & $150(3.7)$ & 0.13 \\
\hline Platelet inhibitors & $10,405(27.7)$ & $1,407(24.2)$ & 0.08 & $2,836(24.0)$ & $935(23.1)$ & 0.02 \\
\hline Lipid regulating drugs & $5,119(13.6)$ & $1,042(17.9)$ & 0.12 & $1,804(15.3)$ & $694(17.2)$ & 0.05 \\
\hline Antipsychotics & $4,216(11.2)$ & $306(5.3)$ & 0.22 & $642(5.4)$ & $217(5.4)$ & $<0.01$ \\
\hline Antidepressants & $3,500(9.3)$ & $461(7.9)$ & 0.05 & $934(7.9)$ & $294(7.3)$ & 0.02 \\
\hline Antidiabetic drugs & $7,244(19.3)$ & $1,171(20.2)$ & 0.02 & $2,290(19.4)$ & $784(19.4)$ & $<0.01$ \\
\hline Oral corticosteroids & $1,677(4.5)$ & $313(5.4)$ & 0.04 & $506(4.3)$ & $161(4.0)$ & 0.02 \\
\hline Non-steroidal anti-inflammatory drugs & $3,360(8.9)$ & $589(10.1)$ & 0.04 & $1,315(11.1)$ & $325(8.0)$ & 0.10 \\
\hline Proton pump inhibitors & $4,393(11.7)$ & $523(9.0)$ & 0.09 & $1,051(8.9)$ & $344(8.5)$ & 0.01 \\
\hline Beta2 agonists & $3,831(10.2)$ & $488(8.4)$ & 0.06 & $901(7.6)$ & $304(7.5)$ & $<0.01$ \\
\hline Inhaled corticosteroids & $1,633(4.3)$ & $246(4.2)$ & 0.01 & $440(3.7)$ & $145(3.6)$ & 0.01 \\
\hline Immunosuppressant & $40(0.1)$ & $21(0.4)$ & 0.05 & $15(0.1)$ & $5(0.1)$ & $<0.01$ \\
\hline
\end{tabular}

N-BPs nitrogen-containing bisphosphonates

ASD Absolute standardized difference, compared with non-exposed group 
Table 2. Risk of pneumonia in patients treated with N-BPs after hip fracture, compared with no treatment

\begin{tabular}{ccccccc}
\hline \multirow{2}{*}{ Outcome } & Group & $\begin{array}{c}\text { Subject, } \\
\mathrm{n}\end{array}$ & $\begin{array}{c}\text { Event, } \\
\mathrm{n}\end{array}$ & $\begin{array}{c}\text { Mortality / } \\
\text { Incidence rate, per } \\
100 \text { person-years }\end{array}$ & $\begin{array}{c}\text { Hazard ratio }^{\mathrm{b}} \\
(95 \% \mathrm{CI})\end{array}$ & $\mathrm{P}$ \\
\hline \multirow{2}{*}{$\begin{array}{c}\text { Incident } \\
\text { pneumonia }\end{array}$} & Non-exposed & 11,802 & 3,595 & $9.0(8.7-9.3)$ & 1 & - \\
& All N-BPs & 4,041 & 977 & $6.9(6.5-7.3)$ & $0.76(0.70-0.83)$ & $<0.001$ \\
& Alendronate & 3,298 & 732 & $6.8(6.3-7.3)$ & $0.74(0.67-0.81)$ & $<0.001$ \\
\hline \multirow{2}{*}{$\begin{array}{c}\text { Pneumonia } \\
\text { mortality }\end{array}$} & Non-exposed & 11,802 & 1,550 & $3.5(3.3-3.7)$ & 1 & - \\
& All N-BPs & 4,041 & 354 & $2.3(2-2.5)$ & $0.65(0.56-0.75)$ & $<0.001$ \\
& Alendronate & 3,298 & 270 & $2.3(2-2.6)$ & $0.63(0.54-0.74)$ & $<0.001$ \\
\hline
\end{tabular}

a N-BPs included alendronate, ibandronate, zoledronate, and risedronate

b Models were adjusted for imbalanced variables (year of index date, frequency of in-patients admission, use of anticoagulants and nonsteroidal anti-inflammatory drugs in the past 180 days) 
Table 3. Risk of pneumonia in patients treated with N-BPs after hip fracture, compared with non-N-BP anti-osteoporosis medications

\begin{tabular}{ccccccc}
\hline \multirow{2}{*}{ Outcome } & Group & $\begin{array}{c}\text { Subject, } \\
\mathrm{n}\end{array}$ & $\begin{array}{c}\text { Event, } \\
\mathrm{n}\end{array}$ & $\begin{array}{c}\text { Mortality / } \\
\text { Incidence rate, per } \\
100 \text { person-years }\end{array}$ & $\begin{array}{c}\text { Hazard ratio } \\
(95 \% \mathrm{CI})\end{array}$ & $\mathrm{P}$ \\
\hline \multirow{2}{*}{ Incident } & Non-N-BPs $^{\mathrm{b}}$ & 507 & 161 & $14(11.9-16.3)$ & 1 & 1 \\
pneumonia & All N-BPs $^{\mathrm{a}}$ & 1,284 & 366 & $8.5(7.7-9.4)$ & $0.68(0.53-0.87)$ & 0.002 \\
& Alendronate & 1,009 & 256 & $8.3(7.3-9.3)$ & $0.67(0.52-0.87)$ & 0.003 \\
\hline \multirow{2}{*}{$\begin{array}{c}\text { Pneumonia } \\
\text { mortality }\end{array}$} & Non-N-BPs & 507 & 75 & $5.6(4.4-7)$ & 1 & 1 \\
& All N-BPs & 1,284 & 139 & $2.9(2.4-3.4)$ & $0.60(0.41-0.89)$ & 0.01 \\
\hline
\end{tabular}

a. N-BPs included alendronate, ibandronate, risedronate, and zoledronate

b. Non-N-BP included denosumab, raloxifene, salcatonin, strontium ranelate, and teriparatide 
54,047 patients aged $\geq 50$ admitted via emergency room with a new diagnosis of hip fracture from Jan 1 2005 to Dec 312015.

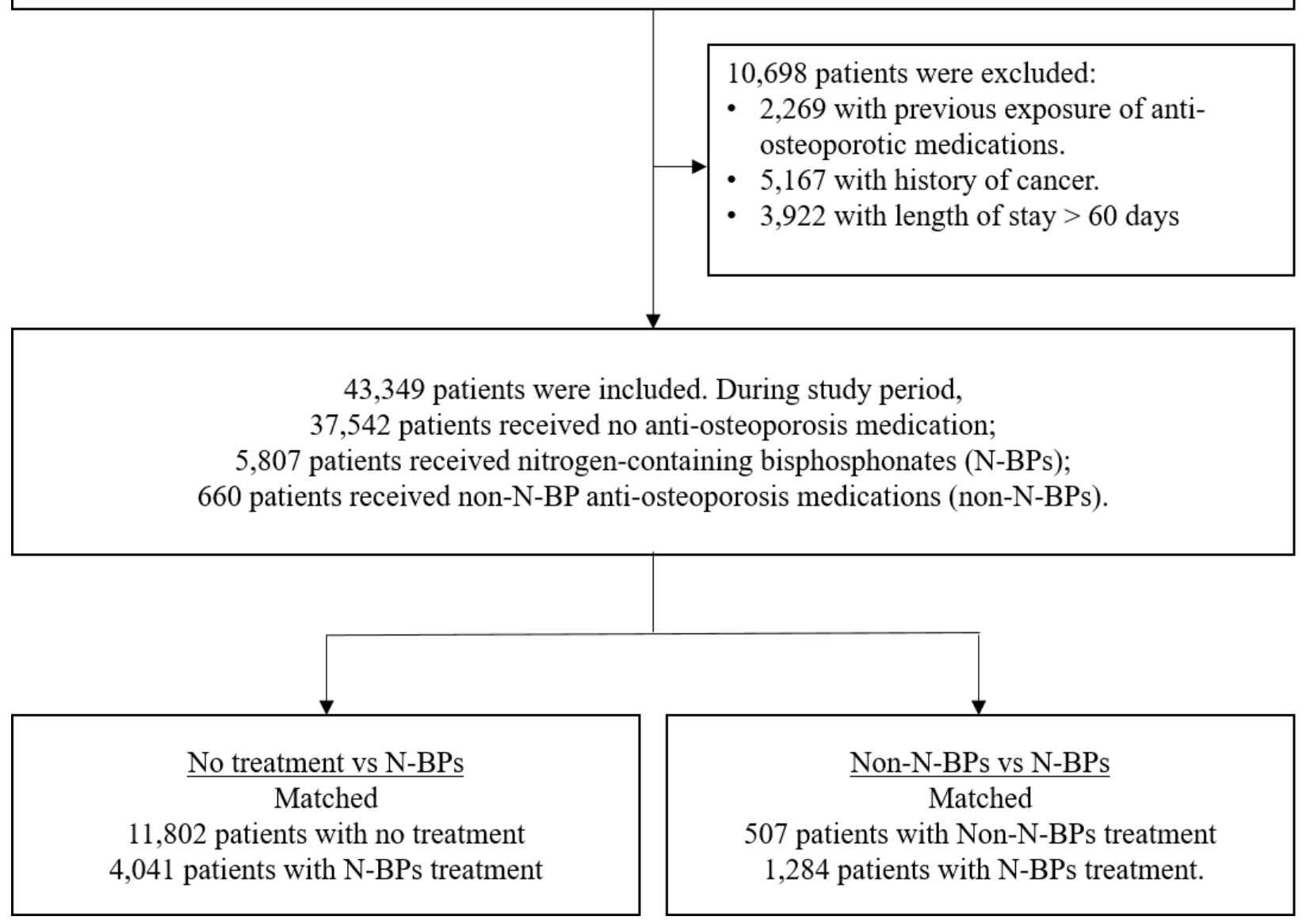

Figure 1. Screening flow chart of the study cohort 
a)

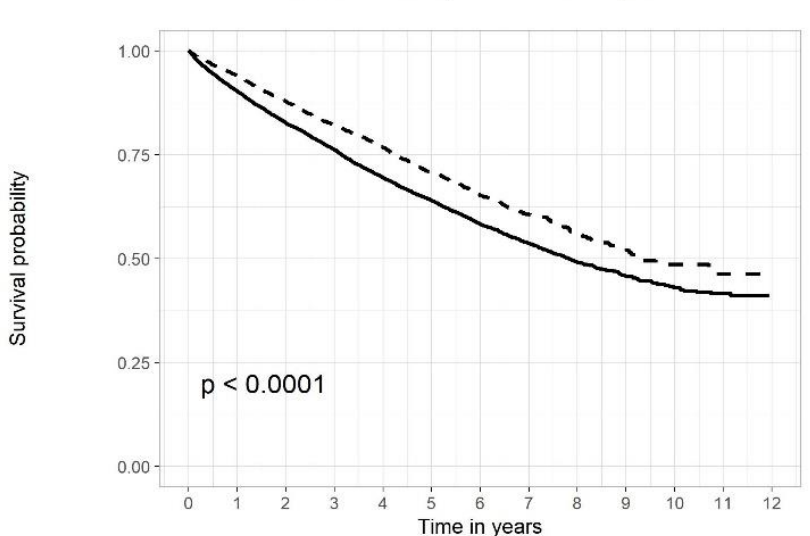

Number at risk

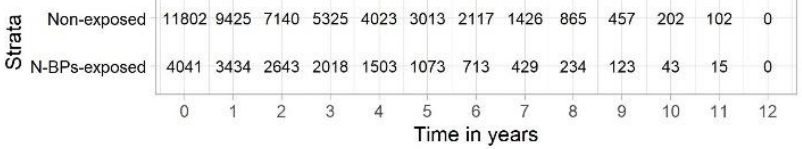

b)

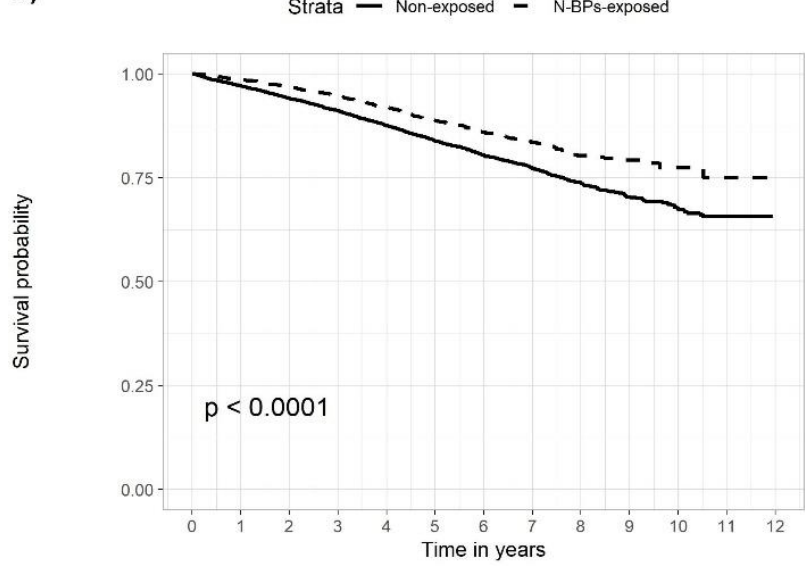

Number at risk

\begin{tabular}{l|l|llllllllllllll} 
& Non-exposed & 11802 & 9998 & 7887 & 6052 & 4696 & 3566 & 2553 & 1733 & 1071 & 567 & 247 & 117 & 0
\end{tabular} \begin{tabular}{l|l|lllllllllllll} 
ஸे N-BPs-exposed & 4041 & 3575 & 2863 & 2246 & 1714 & 1263 & 855 & 527 & 288 & 144 & 51 & 17 & 0
\end{tabular} $\begin{array}{lllllllllllll}0 & 1 & 2 & 3 & 4 & \begin{array}{c}5 \\ \text { Time in years }\end{array} & 8 & 9 & 10 & 11 & 12\end{array}$

Figure 2. Kaplan-Meier curves comparing treatment groups for a) incident pneumonia and b) pneumonia mortality. (p-value tested with stratified log-rank test on matched pairs) 